\title{
Das Schwierigste kommt noch
}

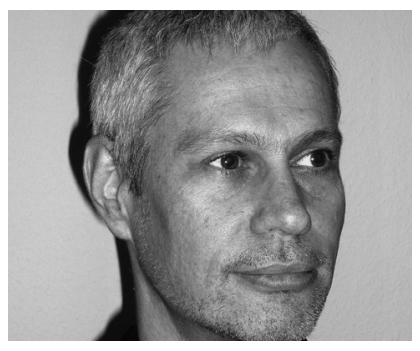

\section{VON CLAUS BÖLICKE}

Claus Bölicke ist Krankenpfleger und Diplom-Pflegewirt (FH). Er arbeitet als Referent für Altenpflege beim Arbeiterwohlfahrt Bundesverband e. V., wo er die Reform der Pflegeversicherung für den Bundesverband begleitet und die Arbeiterwohlfahrt in den Verhandlungen zur Umsetzung des Pflege-Weiterentwicklungsgesetzes vertreten hat.

E-Mail claus.boelicke@awo.org
Die in der letzten Legislaturperiode begonnene Reform der Pflegeversicherung zeigt erste Früchte. Gleichwohl sind die schwierigsten Hindernisse noch nicht beseitigt. Dies betrifft insbesondere die Finanzierung der Pflege, die Umsetzung eines neuen Pflegebedürftigkeitsbegriffs und die Erhöhung der Attraktivität einer beruflichen Tätigkeit in der Pflege.

Die Arbeiterwohlfahrt (AWO) als Spitzenverband der Freien Wohlfahrtspflege mit über 900 teil- und vollstationären Einrichtungen, in denen täglich mehr als 70.000 pflegebedürftige Menschen betreut werden sowie ca. 800 ambulanten Diensten und Sozialstationen, hat sich bereits vor der Einführung der Pflegeversicherung für eine sozialversicherungspflichtige und solidarisch finanzierte Absicherung des Pflegerisikos eingesetzt. Die Arbeiterwohlfahrt hat daher die Reform des Gesetzgebers zum Erhalt und zur Weiterentwicklung der Sozialen Pflegeversicherung durch das Pflege-Weiterentwicklungsgesetz (PfWG) begrüßt und aktiv begleitet. Anderthalb Jahre nach Inkrafttreten der Reform ist es an der Zeit eine erste Zwischenbilanz zur Umsetzung der Reform hinsichtlich der wichtigsten erreichten Leistungsverbesserungen für die Versicherten, des Ziels der Verbesserung der Qualität der Pflege und deren Transparenz sowie der Finanzierung der Pflegeversicherung zu ziehen.

$\mathrm{Zu}$ den grundlegenden Leistungsverbesserungen des Pflege-Weiterentwicklungsgesetzes für die Versicherten gehören

- die Anhebung der Leistungsbeträge und Dynamisierung der Leistungen

- die Stärkung von Beratung, Prävention und Rehabilitation sowie

- die Einführung einer Pflegezeit für Angehörige.

Ferner wurde durch die Reform einige spezifische Leistungsbereiche der pflegerischen Versorgung verbessert, dazu zählen

- die Leistungen der Tages- und Nachtpflege

- die Leistungen für Menschen mit Demenz und
- die ärztliche Versorgung von Pflegebedürftigen in Heimen.

\section{Anhebung der Leistungsbeträge und Dynamisierung der Leistungen}

Die Leistungsbeträge sind seit der Einführung der Pflegeversicherung im Jahre 1995 nicht erhöht worden. Bei einer Preissteigerungsrate von bis zu zwei Prozent pro Jahr war die Konsequenz, dass Pflegebedürftige im Jahre 2007 einen deutlich geringeren Leistungsanspruch hatten als Pflegebedürftige im Jahre 1995. Berechnungen haben ergeben, dass die Leistungen inflationsbedingt in diesen Zeitraum um ca. zwölf Prozent abgewertet worden sind.

Die Anpassung der Leistungsbeträge (vgl. Tabelle 1 Seite 25) war ein notwendiger Schritt in Richtung der Wiederherstellung und Sicherung einer qualitativ angemessenen pflegerischen Versorgung. Die weitere Dynamisierung der Leistungen soll erstmalig 2014 geprüft werden und kann dann frühestens 2015 wirksam werden, so dass die Wirksamkeit dieser Regelung abzuwarten bleibt.

\section{Stärkung von Beratung, Prävention und Rehabilitation}

Die Soziale Pflegeversicherung geht von der Erkenntnis aus, dass Pflegebedürftigkeit vermieden, hinausgeschoben, überwunden oder gelindert werden kann. Aus diesem Grunde wurde der Beratung von Pflegebedürftigen und ihren Angehörigen sowie der Prävention und Rehabilitation dem Gesetz bisher schon Vorrang eingeräumt. Mit dem Pflege-Weiterentwicklungsgesetz wurde dieser bisher eher theoretische Anspruch nun auch praktisch besser untermauert. Zum einen wurden für die Pflegeberatung mit den Pflegestützpunkten und dem Anspruch auf unterstützendes Fallmanagement neue Grundlagen geschaffen.

Für pflegebedürftige Menschen in Heimen kann die Neuregelung zur Rehabilitation von Vorteil sein. Wenn durch entsprechende Maßnahmen eine niedrigere Pflegestufe für einen Versicherten erreicht 
wird, kann die Einrichtung nun einmalig 1.536 Euro pro Pflegebedürftigen als Ausgleich für die dann niedrigere Vergütung erhalten. Dies steuert der bisherigen finanziellen Schlechterstellung der Pflegeeinrichtung durch erfolgreiche Rehabilitationsmaßnahmen entgegen.

Ob diese Regelungen zur Stärkung von Beratung, Prävention und Rehabilitation tatsächlich dazu führen, dass pflegebedürftigen Menschen besser beraten werden und ihnen auch der Zugang zu präventiven und rehabilitativen Maßnahmen nicht mehr versperrt ist, bleibt abzuwarten. Insbesondere die Ansiedelung der Pflegestützpunkte bei den Pflegekassen, lässt Zweifel an einer unabhängigen Beratung aufkommen. Eindeutige Erkenntnisse liegen allerdings zu diesem Themenkomplex noch nicht vor.

\section{Pflegezeit für Angehörige}

Von den insgesamt circa zwei Millionen Menschen, die derzeit Leistungen der Pflegeversicherung erhalten, werden derzeit rund eine Million Menschen alleine von Angehörigen oder sonstigen Pflegeperso- nen in der häuslichen Umgebung betreut. Ein entscheidender Punkt bei der Pflege von Angehörigen ist die Arbeitsplatzsicherheit der Pflegepersonen, die mit dem PflegeWeiterentwicklungsgesetz durch den Anspruch auf Pflegezeit verbessert worden.

Allerdings nur, um kurzfristig auftretende Probleme in einer konkreten Pflegesituation lösen zu können. Die länger anhaltende Pflege von Angehörigen wird dadurch nur bedingt erleichtert. Da die Pflegezeit auch nicht finanziell abgesichert ist, wird dieser Anspruch tatsächlich bisher kaum genutzt.

\section{Leistungen zur Tages- und Nachtpflege}

Die Inanspruchnahme von Tages- und Nachtpflege hatte bisher ein eher geringes Ausmaß, da diese Leistungen in der Regel im häuslichen Bereich zusätzlich in Anspruch genommen wurden und entsprechend durch den Versicherten selbst finanziert werden mussten. Mit dem Pflege-Weiterentwicklungsgesetz ist die teilstationäre Pflege nun zu einem eigenen Leistungsbereich aufgewertet worden. Neben dem voll- en Anspruch auf Tages- und Nachtpflege besteht noch ein hälftiger Anspruch auf die jeweilige ambulante Pflegesachleistung für die weiterhin zu Hause notwendige Pflege.

Die Aufwertung der teilstationären Pflege kann zu einer wahrnehmbaren Entlastung von häuslichen Pflegesituationen führen und scheint zu einer langsam steigenden Nachfrage in diesem Sektor zu führen. Sowohl das langsam steigende Inanspruchnahme-Verhalten als auch der leichte Ausbau der Versorgungsstrukturen und das Interesse von Trägern hieran, scheint dies zu bestätigen, wenn gleich auch regional unterschiedlich stark ausgeprägt.

\section{Leistungen für Menschen mit Demenz}

Die somatische Ausrichtung des Pflegebedürftigkeitsbegriffs der Pflegeversicherung führt bei der Feststellung des Pflegebedarfs dazu, dass psychosoziale Betreuung, Tagesstrukturierung sowie allgemeine Beaufsichtigung Demenzerkrankter nicht berücksichtigt werden. Mit Pflege-Weiterentwicklungsgesetz sind die Ansprüche an

\section{Altenhilfe in der Freien Wohlfahrtspflege}
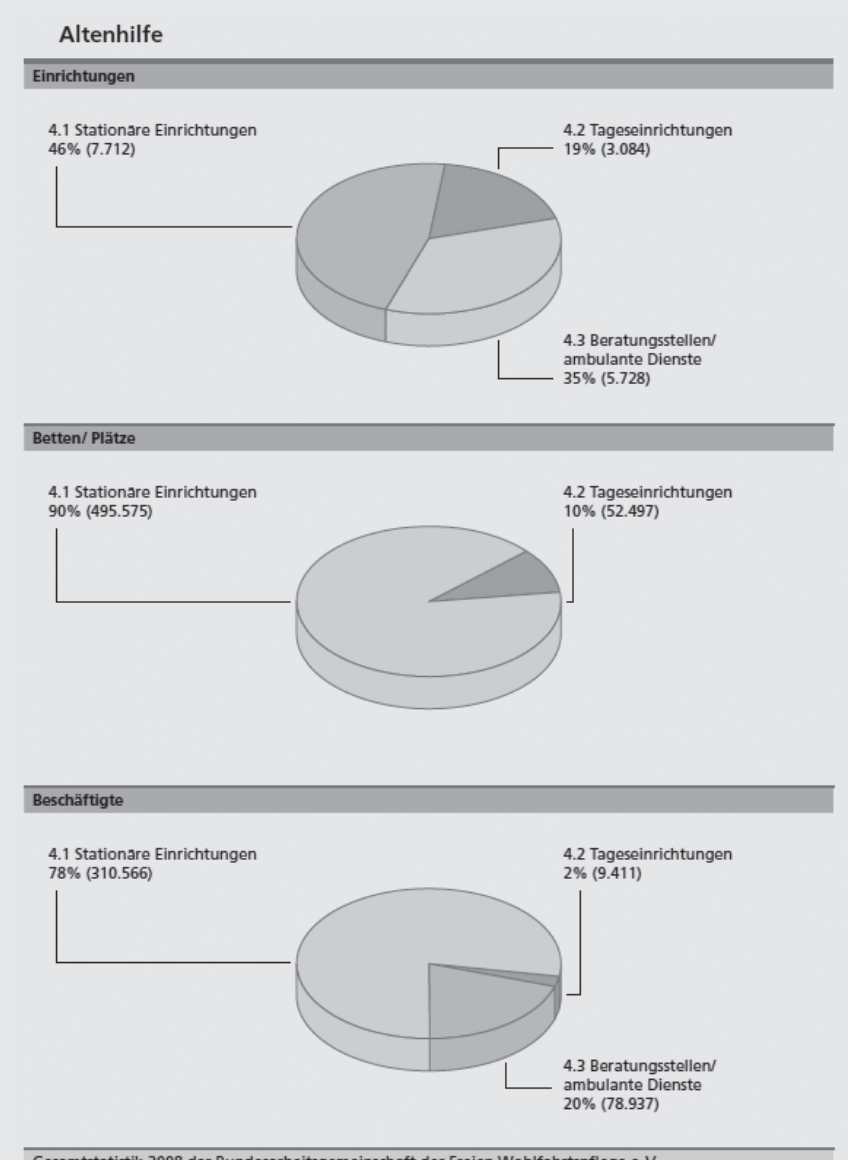

In der Altenhilfe stellen die Wohlfahrtsverbände nach der Gesamtstatistik 2008 bundesweit insgesamt 16.524 Einrichtungen und Dienste bereit. Im Vergleich zur letzen Erhebung aus dem Jahr 2004 ist eine leichte Erhöhung der Zahl der Einrichtungen und Dienste um vier Prozent zu erkennen. Die Altenhilfe zählt mit ihren über 398.000 (2004: ca. 367.000) Mitarbeiterinnen und Mitarbeitern neben der Jugendhilfe und der Gesundheitshilfe zu den größeren Arbeitsbereichen der Freien Wohlfahrtspflege. Die Quote der Teilzeitbeschäftigen in diesem Bereich hat sich von 55 Prozent in 2004 auf 61 Prozent aller Mitarbeiterinnen und Mitarbeiter in 2008 erhöht. 47 Prozent (2004: 44 Prozent) aller Einrichtungen und Dienste der Altenhilfe befinden sich im stationären Bereich. In ihnen werden knapp eine halbe Million Menschen betreut. Hierbei kommt den vollstationären Altenpflegeeinrichtungen (337.330 Betten) und den Seniorenwohnungen einschließlich betreutem Wohnen (144.349 Betten/ Plätze) ein besonderes Gewicht zu. Die Beratungsstellen und ambulanten Dienste konnten ihren Anteil an den Einrichtungen und Diensten im Bereich der Altenhilfe leicht von 33 Prozent in 2004 auf 34 Prozent in 2008 steigern. Darunter befinden sich 2.895 ambulante Pflegedienste und Sozialstationen.

Quelle: Bundesarbeitsgemeinschaft der Freien Wohlfahrtspflege e. V.: Einrichtungen und Dienste der Freien Wohlfahrtspflege. Gesamtstatistik 2008. Berlin 2009. Die Gesamtstatistik 2008 ist als PDF-Dokument auf der Website der Bundesarbeitsgemeinschaft der Freien Wohlfahrtspflege verfügbar. Eine gedruckte Broschüre kann kostenlos bei der Berliner Geschäftsstelle angefordert werden: Bundesarbeitsgemeinschaft der Freien Wohlfahrtspflege e. $V .$, Oranienburger Straße 13-14, 10178 Berlin, Telefon 030 24089o, Fax03024089134,E-Mailinfo@bag-wohlfahrt.de, Internet http://www.bagfw.de 


\begin{tabular}{|l|l|l|l|l||}
\hline Pflegestufe & seit 1995 & ab 2008 & ab 2010 & ab 2012 \\
\hline Stufe I & 205 & 215 & 225 \\
\hline Stufe II & 410 & 420 & 430 \\
\hline Stufe III & 665 & 675 & 685 & (I40 \\
\hline \hline
\end{tabular}

Tabelle 1: Höhe des Pflegegeldes in Euro

\begin{tabular}{|l|l|l|l|l||}
\hline Pflegestufe & seit 1995 & ab 2008 & ab 2010 & ab 2012 \\
\hline |Stufe I & 384 & 420 & 440 \\
\hline Stufe II & 921 & 980 & 1.040 \\
\hline \hline Stufe III & 1.432 & 1.470 & 1.510 \\
\hline $\begin{array}{l}\text { Stufe III + } \\
\text { Härtefall }\end{array}$ & 1.918 & Keine Änderung & Keine Änderung & Keine Änderung \\
\hline \hline
\end{tabular}

Tabelle 2: Höhe der ambulanten Sachleistungen in Euro

\begin{tabular}{|l|l|l|l|l||}
\hline Pflegestufe & seit 1995 & ab 2008 & ab 2010 & ab 2012 \\
\hline Stufe I & 1.023 & Keine Änderung & Keine Änderung & Keine Änderung \\
\hline Stufe II & 1.279 & Keine Änderung & Keine Änderung & Keine Änderung \\
\hline \hline Stufe III & 1.432 & 1.470 & 1.510 \\
\hline $\begin{array}{l}\text { Stufe III + } \\
\text { Härtefall }\end{array}$ & 1.688 & 1.750 & 1.825 \\
\hline
\end{tabular}

\section{Tabelle 3: Höhe der stationären Sachleistungen in Euro}

zusätzliche Betreuungsleistungen aufgrund kognitiver Einschränkungen von Pflegebedürftigen, die Zuhause versorgt werden, von 460 Euro im Jahr auf bis zu 200 Euro monatlich erhöht worden. Zusätzlich erhalten jetzt auch Personen, die einen Hilfebedarf in der Grundpflege und der hauswirtschaftlichen Versorgung haben, der nicht das Ausmaß der Pflegestufe 1 erreicht, die Möglichkeit, diese zusätzliche Betreuungsleistungen in Anspruch zu nehmen. Für Pflegegebedürftige in Heimen konnte mit dem $\mathbb{8} 87 \mathrm{~b}$ SGB XI eine Verbesserung der Betreuungssituation in stationären Pflegeeinrichtungen durch eine zusätzliche Betreuungskraft nach $\mathbb{\$} 87 \mathrm{~b}$ SGB XI erreicht werden.

Das Pflege-Weiterentwicklungsgesetz hat zweifelsohne zu einer Verbesserung der Betreuung von demenzkranken Menschen beigetragen. Zumindest für den stationären Bereich waren die Umsetzungsregelungen allerdings unnötig bürokratisch aufgebläht und haben damit die Einlösung des neuen Leistungsanspruchs um mindestens ein halbes Jahr verzögert. Wesentlich direkter und schneller wäre es gewesen, den Leistungsanspruch nach $\$ 45$ a SGB XI auf die stationäre Situation zu übertragen.

Die grundsätzliche Problematik der rein somatischen Begutachtung der Pflegebedürftigkeit ist mit diesen neuen Regelungen jedoch nicht verändert worden. Hier ist die Umsetzung des neuen Pflegebedürftigkeitsbegriffs in einer nächsten Reform der Pflegeversicherung abzuwarten.

\section{Ärztliche Versorgung von Pflegebedürftigen in Heimen}

Mit einer Öffnungsklausel soll die ärztliche Versorgung in stationären Pflegeein-
Kassenärztlichen Vereinigungen darlegen, die dann die Aufgabe haben, auf den Abschluss von Kooperationsvereinbarungen hinzuwirken. Erst wenn diese Möglichkeiten nicht umgesetzt werden konnten, ist die Anstellung eines Arztes durch den Heimträ-

Die Kompliziertheit dieser Neuregelung und vor allem auch die Unklarheit der Abrechnung bei solchen Modellen hat bisher eine nennenswerte Anzahl von Einstellungen von Heimärzten verhindert. Insbesondere die fachärztliche Versorgung von Heimbewohnern ist daher nach wie vor defizitär.

\section{Qualität und Transparenz}

Zur Verbesserung der Qualität und deren Transparenz sind mit dem Pflege-Weiterentwicklungsgesetz einige neue Regelungen in Kraft getreten. Es wurde erstmalig im Rahmen der Pflegeversicherung eine Schiedsstelle installiert, die bei Streitigkeiger zulässig. richtungen durch die Beschäftigung durch sogenannte Heimärzte verbessert werden. Die Anstellung eines Heimarztes nach $\mathbb{}$ 119b SGB V ist jedoch nur als Verbesserung der ärztlichen Versorgung der Heimbewohner vorgesehen. Zuvor muss das Heim alle Möglichkeiten von Kooperationsvereinbarungen mit Arztpraxen ausloten und die Versorgungsproblematik gegenüber den

ten zwischen den Vertragsparteien bei Vereinbarungen zur Qualität vermitteln soll. $\mathrm{Zu}$ diesen Vereinbarungen gehören die Grundsätze und Maßstäbe zur Qualität und Qualitätssicherung nach $\mathbb{S} 113$ (ehemals $\mathbb{S} 80$ ) SGB XI sowie die neu im Gesetz verankerten Pflege-Transparenzvereinbarungen nach $\$ 115$ SGB XI und die Expertenstandards nach $\mathbb{1 1 3}$ a SGB XI.

Die Regelungen zur Qualität und Transparenz sind grundsätzlich begrüßenswert und stellen besonders in der öffentlichen Qualitätsberichterstattung einen wirklichen Meilenstein dar. Dies geht jedoch mit einem immensen Ausbau externer Kontrollen einher. Externe Kontrollen sind zwar ein wichtiger Baustein der Qualitätssicherung, als Folge werden hier aber unnötig knappe finanzielle und personelle Ressourcen in der Pflege für Bürokratie gebunden, statt nachhaltig in die Entwicklung der Qualität vor Ort und die Stärkung eines nachhaltigen internen Qualitätsmanagements zu investieren.

\section{Finanzierung}

Mit der Erhöhung der Beitragssätze durch das Pflege-Weiterentwicklungsgesetz konnte kurzfristig eine Stabilisierung der finanziellen Ausstattung der Pflegeversicherung erreicht werden, die längst fällige Leistungsverbesserungen und - erstmalig seit
Einführung der Versicherung - auch eine Dynamisierung der Leistungsentgelte möglich gemacht hat. $\mathrm{Ob}$ diese Maßnahmen aber auch zu einer langfristigen Stabilisierung der Finanzen der Pflegeversicherung führen, wird allgemein eher bezweifelt werden.

Langfristig hält die Arbeiterwohlfahrt daher weitere Schritte zur finanziellen Absicherung der Pflegeversicherung im Sinne der Umwandlung in eine Bürgerversicherung für notwendig.

\section{Fazit}

Neben den aufgeführten Änderungen wurden noch eine Reihe weiterer Neuerungen eingeführt, die jedoch praktisch bisher kaum Relevanz entfaltet haben oder noch der Umsetzung harren. Dazu gehören beispielsweise die Befristung von Pflegestufen, die Möglichkeit Gesamtversorgungsverträge zu schließen, der Komplex der ortsüblichen Vergütung und des externen Ver- 


\title{
Die Meilensteine der Pflegeversicherung
}

\author{
1995 Pflege-Versicherungsgesetz, Gesetz zur Änderung des Gesetzes zur \\ sozialen Absicherung des Risikos der Pflegebedürftigkeit \\ 1996 1. SGB XI-ÄndG \\ 1998 Gesetz zur sozialen Absicherung flexibler Arbeitszeitregelungen, \\ 3. SGB XI-ÄndG \\ 1999 Gesetz zur Neuregelung der geringfügigen Beschäftigungsverhältnisse, \\ 4. SGB XI-ÄndG \\ 2000 Haushaltssanierungsgesetz, GKV-Gesundheitsreform 2000 \\ 2002 Pflege-Qualitätssicherungsgesetz (PqsG), \\ Pflegeleistungs-Ergänzungsgesetz (PflEG) \\ 2005 Kinder-Berücksichtigungsgesetz (KiBG), \\ Verwaltungsvereinfachungsgesetz \\ 2007 Gesetz zur Stärkung des Wettbewerbs in der GKV (GKV-WSG) \\ 2008 Pflege-Weiterentwicklungsgesetz
}

gleichs (wie auch der Umsetzung von Mindestlöhnen) oder die Kompetenzerweiterung für Gesundheitsberufe.

Mit dem Pflege-Weiterentwicklungsgesetz hat der Gesetzgeber dennoch einen wichtigen Schritt in Richtung der Verbesserung der Versorgungssituation, insbeson- dere bisher leistungsrechtlich benachteiligter Personengruppen getan sowie den Grundstein für eine fundierte Information über das Leistungsgeschehen in der Pflege gelegt. Damit konnten jedoch bei weitem nicht alle anstehenden Probleme im Zusammenhang mit der Pflege (-versicherung) gelöst werden, so dass weitere Reformschritte unausweichlich sind. Als wichtigste Punkte sind hierbei herauszuheben

- die nachhaltige Absicherung der Finanzierung der Pflege

- die Umsetzung des neuen Pflegebedürftigkeitsbegriffs

- die Stärkung einer nachhaltigen internen Qualitätsentwicklung und

- die angemessene Vergütung von Leistungen.

Eine weitere Herausforderung in der Pflege wird in den nächsten Jahren der zunehmende Mangel an Fachkräften, aber auch an Hilfskräften, darstellen. Die geringe Attraktivität des Berufsfelds aufgrund schwieriger Arbeitsbedingungen bei hohen Anforderungen an Persönlichkeit und Qualifikation bei teils mäßiger Bezahlung wird die Pflege vor enorme Probleme im Kampf um Mitarbeiter und Auszubildende mit anderen Branchen stellen. In der letzten Legislaturperioden eingeleitete Vorhaben, wie beispielsweise die Einführung eines Mindestlohns in der Pflege, stehen unter der neuen Regierung wieder auf der Kippe. Gleichzeitig ist im Koalitionsvertrag zu dieser Problematik so gut wie keine Aussage zu finden.

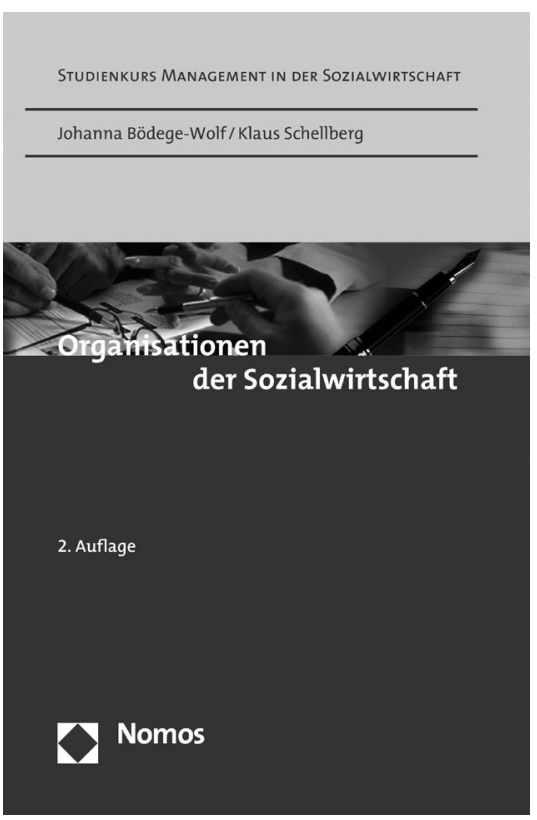

Bitte bestellen Sie im Buchhandel oder versandkostenfrei unter $\bullet$ www.nomos-shop.de

\section{Organisationen der Sozialwirtschaft}

Prof. Dr Johanna Bödege-Wolf und Prof. Dr. Klaus Schellberg

2. Auflage 2010, ca. 180 S., brosch., ca. 19,80 €, ISBN 978-3-8329-5206-8

(Studienkurs Management in der Sozialwirtschaft)

Erscheint ca. Mai 2010

Der Band ist für ein fundiertes Verständnis der verschiedenen Organisationen des Sozialsektors und ihrer Managementaufgaben unerlässlich. Die Autoren liefern einen aktuellen Überblick über Strukturen, Rechtsformen, ideelle Grundlagen, Entwicklungslinien, Formen der Zusammenarbeit und Managementaufgaben der freien und öffentlichen Träger sowie der Dienstleistungsunternehmen. 